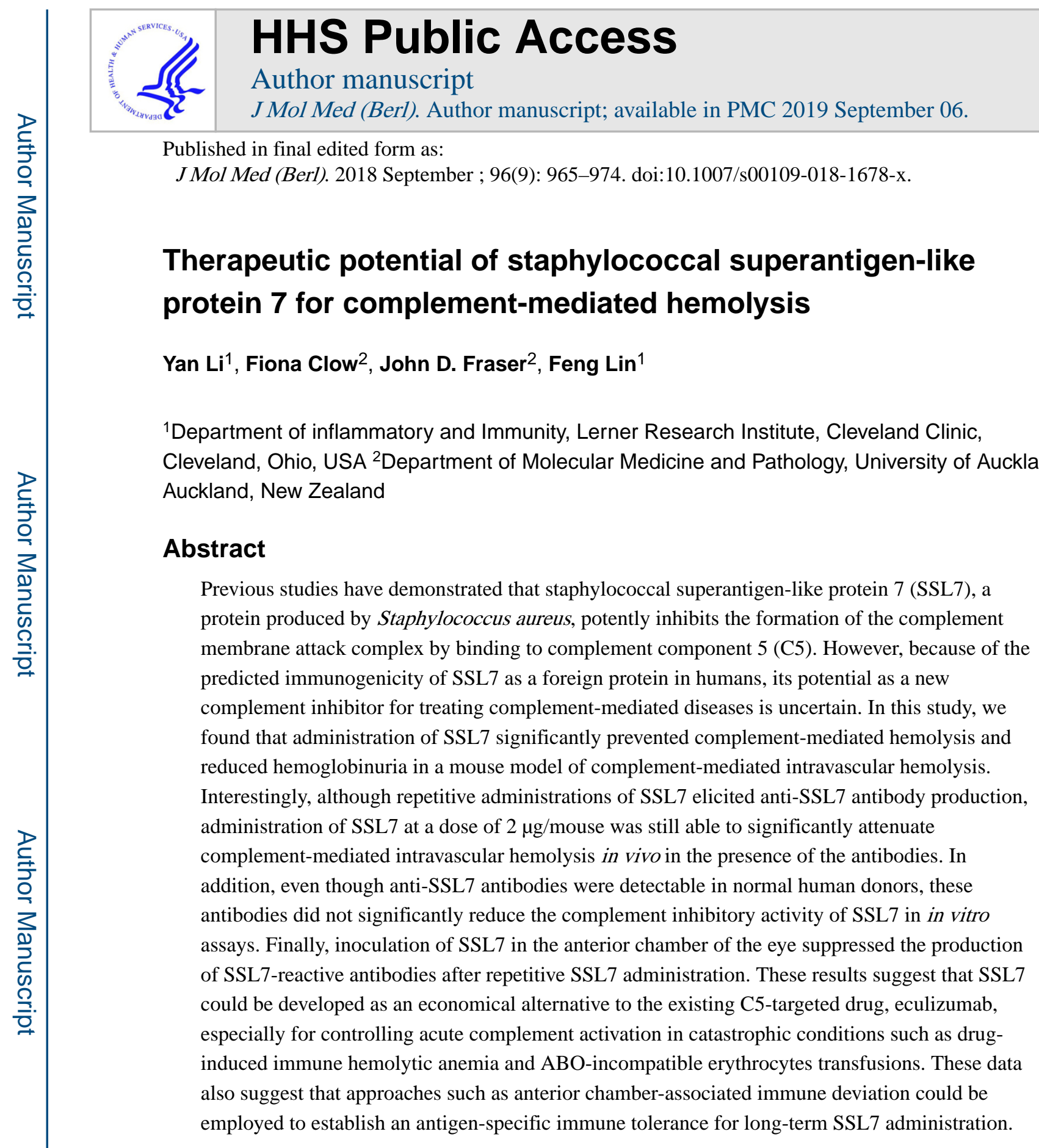

\title{
Introduction
}

Complement is an important element of the innate immune system whose primary role is to protect the host from infections. After initiation through one of the three major complement activation pathways, i.e., the classical, alternative and lectin pathway, activated complement

\footnotetext{
Correspondence to: Feng Lin, Ph.D., Department of Immunology, Cleveland Clinic, Cleveland, OH 44106, Linf2@ ccf.org. Authorship Contributions

YL and FC did the experiments, FL and JDF designed and supervised the studies. FL wrote the manuscript and YL, FC and JDF contributed to the manuscript editing.

Disclosure of Conflicts of Interest

The authors declare no conflicts of interest.
} 
assembles membrane attack complexes (MACs) on the target cells to form "pores", leading to osmotic lysis and killing of the invading pathogens[1].

Although it is critical in fighting infection, activated complement cannot distinguish selfcells from foreign targets. The protection of self-cells from bystander activated complementmediated attack depends largely on the activity of cell surface complement regulatory molecules such as CD55 and CD59[2], which are deficient on some erythrocytes of paroxysmal nocturnal hemoglobinuria $(\mathrm{PNH})$ patients due to a somatic pig $A$ gene mutation[3]. MAC-mediated hemolysis in these patients leads to hemoglobinuria and anemia[4]. In some cold agglutinin disease (CAD) patients, complement is activated on erythrocytes by anti-erythrocyte surface antigen autoantibodies, overwhelming the protection of cell surface complement regulators, which also leads to hemolytic anemia[5, 6]. Consequently, eculizumab, a humanized anti-C5 mAb that inhibits the formation of MAC, has been developed and approved for treating PNH patients[7, 8]. It is also effective in treating CAD patients $[9,10]$. Although highly effective, eculizumab is the most expensive drug on the market, costing about half million dollars a year per patient[11]. The development of economical and effective alternative $\mathrm{C} 5$ inhibitors is of great importance and urgency.

During evolution, pathogens developed multiple mechanisms to protect themselves from the attacks of the host immune system, especially those from the complement system. Coversin, also known as broad-acting $\mathrm{C}$ inhibitory protein $(\mathrm{OmCI})$, is a native protein produced by the soft tick Ornithodoros moubata. It binds to C5 and inhibits MAC-mediated hemolysis[12] This protein has also been found effective in treating experimental autoimmune myasthenia gravis[13], in which massive MAC formation in the neuromuscular junctions damages the endplates, leading to disease[14, 15]. Because of its potent C5 inhibitory activity, low molecular weight $(\sim 17 \mathrm{KDa})$ and minimal observed immunogenicity, coversin is currently being evaluated in a Phase II clinical trial as a potential alternative of eculizumab for treating PNH (NCT02591862). In addition to coversin, staphylococcal superantigen-like protein 7 (SSL7), a small protein produced by the opportunistic bacterial pathogen Staphylococcus aureus, is also found to be able to bind to C5, thereby inhibiting MAC formation[16-18]. Because of its potent complement inhibitory activity, SSL7 could also be developed as a new agent for treating complement-mediated diseases such as PNH and CAD. However, partially due to its predicted immunogenicity as a foreign protein, the potential of SSL7 as a new complement inhibiting therapeutic has been neglected. It has not been previously evaluated in vivo for its efficacy in inhibiting complement-mediated hemolysis either.

In this project, we tested the efficacy of SSL7 in treating a mouse model of complementmediated intravascular hemolysis, a clinical sign presented in many disorders including $\mathrm{PNH}, \mathrm{ABO}$-incompatible red blood cell transfusion, and CAD. We also evaluated the immunogenicity of SSL7 by repeatedly injecting SSL7 intraperitoneally by itself, and then measured levels of SSL7-specific antibodies and assessed the impact of these antibodies on SSL7 treatment efficacy thereafter. In addition, we examined the potential effect of preexisting anti-SSL7 antibodies in humans on the complement-inhibiting activity of SSL7. Finally, we investigated a strategy to induce SSL7-specific immune tolerance to minimize the immunogenicity of SSL7 for long-term, repetitive administration. 


\section{Methods and reagents}

\section{Human blood samples}

Sera from normal human donors were collected at Emory Eye Center, Emory University between December 16, 2009, and March 21, 2010[19]. Informed consent was obtained from all subjects. Subjects were excluded if they were younger than 18, or were older than 90 years of age. Donors with a suspected diagnosis of infection or chronic diseases were excluded as well. The sample collection procedure was approved by the Institutional Review Board (IRB) at the Emory University[19].

\section{Mice and other reagents}

C57BL/6 Wild-type (WT) mice were ordered from the Jackson Laboratory (Bar Harbor, $\mathrm{ME}$ ) and maintained under pathogen-free conditions in the animal facility of Lerner Research Institute, Cleveland Clinic. All procedures involving mice were approved by the Institutional Animal Care and Use Committee of Cleveland Clinic. Recombinant SSL7 and SSL7-C5, a SSL7 mutant lacking C5 binding activity were overexpressed in E.coli and purified following the protocol described in previous reports[16, 20]. Pooled normal human serum (NHS) as the source of complement was purchased from Innovative Research (Novi, MI).

\section{In vitro Complement-Mediated Hemolysis Assay}

Complement-mediated hemolysis assays were done as reported previously[21, 22]. In brief, antibody-sensitized sheep erythrocytes $\left(\mathrm{E}^{\mathrm{shA}}\right)$ were incubated with 5\% NHS in the GVB++ buffer (5 mM Barbital, $145 \mathrm{mM} \mathrm{NaCl}, 0.5 \mathrm{mM} \mathrm{MgCl} 2,0.15 \mathrm{mM} \mathrm{CaCl}_{2}$, and $0.1 \%$ Gelatin, $\mathrm{pH}$ 7.4) in a total volume of $100 \mu \mathrm{L}$ in the presence of different concentrations of SSL7 or SSL7-C5. For a negative control, $5 \mathrm{mM}$ EDTA was added. After incubation at $37^{\circ} \mathrm{C}$ for 5 min, samples were centrifuged, and the absorbance of the supernatant was measured at 414 $\mathrm{nm}\left(\mathrm{OD}_{414}\right)$ using a microtiter plate reader (Molecular Devices, Sunnyvale, CA). The following equation was used to calculate the percentage of hemolysis in each sample: hemolysis rate $(\%)=[(\mathrm{A}-\mathrm{B}) /(\mathrm{C}-\mathrm{B})] \times 100 \% . \mathrm{A}=$ the $\mathrm{OD}_{414}$ reading of sample in $\mathrm{GVB}+$ ,$+ \mathrm{B}=$ the $\mathrm{OD}_{414}$ reading of sample in $\mathrm{GVB}++$ with $5 \mathrm{mM}$ EDTA, and $\mathrm{C}=$ the $\mathrm{OD}_{414}$ reading of maximum hemolysis induced by $\mathrm{H}_{2} \mathrm{O}$.

\section{In vivo Complement-Mediated Intravascular Hemolysis and Hemoglobinuria.}

Complement-induced intravascular hemolytic anemia was initiated by intravenouslyinjecting $200 \mu \mathrm{l}$ of NHS into a WT mouse following a previously published protocol[22, 23]. For treatment studies, each mouse received $2 \mu \mathrm{g}$ of SSL7 or SSL7-C5 by intraperitoneal injection $1 \mathrm{hr}$ before NHS administration. Thirty min later, blood and urine samples were collected for analyses. For the hematocrit reduction measurements, hematocrit capillary tubes treated with $4 \%$ Sodium Citrate were filled with blood, then sealed at one end before being placed in the microhematocrit centrifuge for spinning at 12,000 rpm for 2 minutes. Hematocrit reductions were calculated using the following equation: hemolysis reduction $(\%)=[(\mathrm{A}-\mathrm{B}) / \mathrm{B}] \times 100 \%$. A $=$ hematocrit readings of samples after NHS injection, $\mathrm{B}=$ hematocrit readings of samples before NHS injection. Intravascular hemolysis was 
quantitated by OD reading at $414 \mathrm{~nm}$ after diluting the plasma at 1:100 ratio using PBS in a volume of $100 \mu \mathrm{l}$ whilst hemoglobinuria was assessed by OD reading at $414 \mathrm{~nm}$ after diluting the urine at 1:10 ratio using PBS in a volume of $100 \mu$.

\section{Mouse blood anti-SSL7 antibody Measurement by ELISA.}

Each mouse was injected intraperitoneally with $2 \mu \mathrm{g}$ of SSL7 in PBS once every other week for 6 weeks. Then blood was collected from the mouse tail vein, and the serum anti-SSL7 IgG levels were measured by ELISA. Briefly, an ELISA plate was coated with $5 \mu \mathrm{g} / \mathrm{mL}$ purified SSL7. After blocking, 1:100 diluted serum samples were added to the plate and incubated for $2 \mathrm{~h}$. After washing, HRP-anti-mouse IgG was added. After developing, levels of anti-SSL7 IgG in the blood were assessed by measuring absorbance at $450 \mathrm{~nm}$.

\section{Human blood anti-SSL7 antibody Measurement by ELISA.}

Fifty serum samples from the healthy donor group were randomly selected from a previous study[19] for the measurement of pre-existing anti-SSL7 antibodies. To do this, an ELISA plate was coated with $5 \mu \mathrm{g} / \mathrm{mL}$ purified SSL7. After blocking, 1:1000 diluted human serum samples were added to the plate and incubated for $2 \mathrm{~h}$. After washing, HRP-mouse antihuman $\operatorname{IgG}(2 \mu \mathrm{g} / \mathrm{ml}$, Jackson ImmunoResearch, PA) was added as a detective antibody. Levels of anti-SSL7 IgG in the blood were assessed by measuring absorbance at $450 \mathrm{~nm}$

\section{Complement inhibitory activity assay of SSL7 using heat-inactivated human sera}

Serum samples containing high or low levels of anti-SSL7 IgGs as determined in the abovedescribed ELISA were selected and first incubated at $56^{\circ} \mathrm{C}$ for $30 \mathrm{~min}$ to inactivate the endogenous complement. After this, individual serum (undiluted) was incubated with 1 or 5 $\mu \mathrm{g} / \mathrm{ml}$ of SSL7 in a volume of $22 \mu \mathrm{l}$ on ice for $30 \mathrm{~min}$. The complement inhibitory activities of SSL7 in these mixtures were tested using the same in vitro complement-mediated hemolysis assay using $5 \%$ NHS as the source of complement as described above.

\section{Painting purified SSL7 onto mesenchymal stem cells (MSCs)}

Primary human MSCs were isolated from bone marrow from healthy donors at the MSC Core Facility at Case Western Reserve University as described previously[24, 25], and SSL7 was painted on the surface of MSCs following our previously reported protocol[24, 25]. After painting, cells were washed with PBS and used for experiments. The SSL7 painting efficiency was evaluated by flow cytometry after staining the painted cells with an anti-SSL7 antibody.

\section{Complement inhibitory activity assay of painted SSL7 on MSCs}

To determine the effect of anti-SSL7 IgGs existing in human sera on the complement inhibitory activity of SSL7, we test efficacies of the painted SSL7 on the surface of MSCs in protecting the cells from complement-mediated cell damage using a 2', 7'-bis-(2carboxyethyl)-5-(and-6)-carboxyfluorescein (BCECF) leakage-based cell damage assay[24, 25]. To do this, $2 \times 10^{4}$ of SSL7-painted MSCs were first loaded with $5 \mu \mathrm{M}$ BCECF-AM (ThermoFisher, Waltham,MA), then incubated with $30 \%$ of the same selected sera containing high or low levels of anti-SSL7 antibodies in PBS with 5 mM EDTA buffer for 
30 min on ice to allow the anti-SSL7 antibodies to bind without activating complement. Then cells were washed and incubated for 30 minutes at $37{ }^{\circ} \mathrm{C}$ with $30 \%$ normal human serum in $100 \mu \mathrm{l}$ of GVB-Mg-EGTA buffer (0.1\% Gelatin, $5 \mathrm{mM}$ Barbital, $145 \mathrm{mM} \mathrm{NaCl}$, $0.5 \mathrm{mM} \mathrm{MgCl} 2$, and $10 \mathrm{mM}$ EGTA, $\mathrm{pH}$ 7.3) to allow complement activation through the alternative pathway. The inhibitory activity of the SSL7 on the MSC surface was then assessed by measuring levels of BCECF leaked into the supernatants as a result of complement-mediated cell damage using a fluorescence PerkinElmer Victor3 plate reader (PerkinElmer, Waltham, MA) with excitation and emission wavelengths of $485 \mathrm{~nm}$ and 538 $\mathrm{nm}$, respectively following a previously published protocol[24, 25].

\section{Anterior chamber (AC) of the eye injection of SSL7}

Injection of $\mathrm{AC}$ of the eye was done following a protocol previously reported[26, 27]. For all AC injections, mice were first anesthetized with isoflurane using a mouse anesthetization machine (VetEquip, Pleasanton, CA). The cornea was then punctured by inserting a 33gauge needle directly above the pupil and parallel to the iris. Aqueous humor was allowed to flow out and removed by cotton tips. One $\mu \mathrm{g}$ of SSL7 in 5 $\mu 1$ of PBS or the same volume of PBS was injected into the AC with a microsyringe fitted with a 33-gauge blunt needle. The air bubble seals the corneal puncture and prevents leaking of liquids. Two weeks after anterior chamber inoculation, each of these mice was injected with $2 \mu \mathrm{g}$ of SSL7 intraperitoneally once every week for 6 weeks before serum levels of anti-SSL7 IgGs were compared by ELISA.

\section{Statistical analysis}

Experiments were repeated at least twice, with data reported as mean \pm SEM. All results were analyzed using the Student's t-test. Probability $(p)$ values less than 0.05 were considered statistically significant.

\section{Results}

\section{SSL7 inhibits complement-mediated hemolysis in vitro}

We first determined the capacity of SSL7 to inhibit complement-mediated hemolysis in vitro. We incubated antibody-sensitized sheep erythrocytes ( $\left.\mathrm{E}^{\mathrm{shA}}\right)$ with 5\% NHS in GVB++ buffer in the presence of different concentrations of SSL7 or the mutant SSL7 that lacks C5 binding activity (SSL7-C5), then analyzed the degree of hemolysis by reading $\mathrm{OD}_{414}$ of the supernatants as a measure of the released hemoglobin. As previously reported that SSL7 inhibits the serum killing of a Gram-negative organism Escherichia coll[16], we found that SSL7, but not the mutant SSL7-C5, significantly inhibited complement-mediated hemolysis in a dose-dependent manner (Fig. 1).

\section{SSL7 treatment reduces complement-mediated intravascular hemolysis and hemoglobinuria in vivo}

Previous studies have demonstrated that injecting NHS into mice induces massive complement-mediated intravascular hemolysis and hemoglobinuria, which serves as a model for complement-mediated hemolytic anemia [22, 23]. To test whether SSL7 can attenuate complement-mediated intravascular hemolysis in vivo, we injected intraperitoneally each 
mouse with $2 \mu \mathrm{g}$ of SSL7 or SSL7-C5, then $1 \mathrm{hr}$ later, intravenously injected them with 200 $\mu \mathrm{l}$ of NHS. After 30 minutes, we bled the mice retro-orbitally to measure the reduction of hematocrit and to assess released hemoglobin levels in the plasma by reading $\mathrm{OD}_{414}$. We also collected urine to read $\mathrm{OD}_{414}$ as a way to measure hemoglobinuria. These assays showed that $\mathrm{OD}_{414}$ readings (hemoglobin levels) of the plasma from mice treated with SSL7 were $0.22 \pm 0.01$, compared to $0.31 \pm 0.01$ from mice treated with the control SSL7-C5 (Fig. 2A). In addition, the hematocrit readings of mice treated with SSL7-C5, the mutant SSL7 with minimal complement inhibitory activity, were reduced by $46.46 \pm 13.6 \%$. Conversely, hematocrit readings were reduced by only $7.82 \pm 6.96 \%$ in mice treated with SSL7 (Fig. 2B). Consistent with both of these blood tests, $\mathrm{OD}_{414}$ readings of the urine samples from mice treated with SSL7 were $0.07 \pm 0.04$, compared to $0.14 \pm 0.02$ in mice treated with the control SSL7-C5 (Fig. 2C). These results clearly demonstrate that at the dose of $2 \mu \mathrm{g} / \mathrm{mouse}$, SSL7 effectively inhibits complement-mediated hemolysis in vivo.

\section{Repetitive administration of SSL7 elicits anti-SSL7 antibody production but does not reduce SSL7 efficacy in treating complement-mediated hemolysis in vivo}

To mimic clinical conditions in which patients might receive multiple treatments, and to evaluate the impact of the predicted immunogenicity of SSL7 on its treatment efficacy, we treated each mouse with $2 \mu \mathrm{g}$ of SSL7 in PBS or PBS alone by intraperitoneal injection once every other week for 6 weeks, collected the plasma samples at weeks 0, 2, 4 and 6, then measured anti-SSL7 IgG levels in these plasma sample by ELISA. These experiments showed that mice receiving repetitive SSL7 administration developed an elevated level of anti-SSL7 IgGs (Fig. 3A), confirming the predicted immunogenicity of SSL7. We then examined the efficacy of intraperitoneal injection of $2 \mu \mathrm{g}$ of SSL7 in these mice in inhibiting complement-mediated hemolysis by measuring plasma hemoglobin levels, hematocrit reduction, as well as the severity of hemoglobinuria. We found that in the presence of antiSSL7 antibodies developed after repetitive SSL7 administration, SSL7 was still able to significantly reduce complement-mediated hemolysis in these mice and there was no significant difference between the mice pre-exposed to SSL7 and developed anti-SSL7 antibodies and those did not (Fig.3B, C\&D).

\section{Pre-existing anti-SSL7 antibodies in humans do not significantly inhibit the complement- inhibitory activity of SSL7}

Staphylococcus aureus is a relatively common pathogen and anti-SSL7 antibodies could develop after infection in humans. To test whether these pre-existing anti-SSL7 antibodies could affect the treatment efficacy of SSL7, we first screened a cohort of already collected human sera for levels of anti-SSL7 IgGs by ELISA. We found that levels of anti-SSL7 antibodies differ significantly in the screened sera (Fig. 4A), suggesting that some donors may have previously had an $S$. aureus infection and thus developed anti-SSL7 antibodies while others did not. We then picked 10 sera with the highest and 10 with lowest levels of anti-SSL7 IgGs for further analysis. To avoid the potential interference of different complement activities in sera from different donors, we first heat-inactivated the endogenous complement, then incubated the sera with different concentrations of SSL7 to allow the antiSSL7 antibodies within to bind to SSL7. We then tested the complement inhibitory activity of SSL7 in the mixtures using the standard hemolysis inhibition assay. As shown in Fig. 4, 
both tested concentrations of SSL7 significantly inhibited complement-mediated hemolysis, and there was no statistically significant difference between samples with SSL7 incubated with heat-inactivated sera containing high or low levels of anti-SSL7 antibodies.

To rigorously evaluate the effect of the pre-existing anti-SSL7 antibodies on the complement inhibitory activity of SSL7, in addition to above experiments using heat-inactivated sera, we also employed another approach to minimize the variables that might be induced by different complement and complement inhibitor activities in sera from different donors. To do this, we first coated SSL7 protein onto MSCs (Fig. 4B), let the anti-SSL7 antibody binds to the painted SSL7, then tested the impacts of anti-SSL7 antibodies in reducing the protective effects of the painted SSL7 in reducing complement-mediated MSC damage using a common source of complement[21]. In brief, we incubated the SSL7-painted MSCs with the individual serum sample in the presence of EDTA to allow the anti-SSL7 antibodies to bind to the SLL7 on the MSC cell surface without activating complement. We then exposed these treated MSCs to a pooled NHS as the common source of complement (no existing anti-SSL7 IgGs, Fig, 4A), and assessed MSC damage. As shown in Fig. 4C, we found no significant difference in MSC damage between the SSL7-painted MSCs incubated with sera containing high titers of anti-SSL7 IgGs and those incubated with sera containing low titers of antiSSL7 IgGs, suggesting that the pre-existing anti-SSL7 antibodies in humans should not have a significant impact on the complement inhibitory activity of SSL7.

\section{Anterior Chamber injection of SSL7 reduces the production of anti- SSL7 antibodies after repetitive administration}

It has been previously established that inoculation of an antigen into the anterior chamber (AC) of the eye establishes antigen-specific immune tolerance[26, 27]. Consequently, we evaluated the possibility that injection of SSL7 in the AC of the mice could reduce the immunogenicity of SSL7 for the future long-term and repetitive administrations. To test this hypothesis, we first injected $1 \mu \mathrm{g}$ of SSL7 or the same volume of PBS into the AC of naïve WT mice, then treated these mice by repetitive intraperitoneal injection of SSL7 every other week for 6 weeks and collected plasma samples each time when SSL7 was given. We then measured levels of SSL7-specific IgGs in the plasma of these mice at different time points by ELISA. We found that again that mice received repetitive intraperitoneal injection of SSL7 developed anti-SSL7 antibodies, but mice with AC injection of SSL7 developed significantly lower levels of SSL7-specific IgGs than mice with AC injection of vehicles (PBS) (Fig. 5A). After inducing complement-mediated hemolysis in these mice by injection of NHS as done in above-described experiments, we treated them with the same amount of SSL7 ( $2 \mu \mathrm{g} / \mathrm{mouse}$ ), followed by the evaluations of their hematocrit reductions, free plasma hemoglobin levels and hemoglobinuria levels. These experiments showed that SSL7 treatment in mice with prior AC injection of SSL7 resulted in a trend of decreased plasma hemoglobin levels (Fig. 5B), hematocrit reduction (Fig. 5C) and hemoglobinuria levels (Fig. 5D) than in mice with prior $\mathrm{AC}$ injection of the vehicle (PBS), although the reductions were not statistically significant. These data suggest that AC injection of SSL7 is effective in reducing the immunogenicity of SSL7 and confirm that anti-SSL7 antibodies generated after repetitive administrations should not have a drastic effect on SSL7 treatment efficacy. 


\section{Discussion}

In this report, we showed evidence, for the first time, that SSL7 effectively inhibited complement-mediated intravascular hemolysis in vivo. We found that although repetitive administration of SSL7 elicited the production of SSL7-specific antibodies, these antibodies did not significantly affect the efficacy of administrated SSL7 in reducing complementmediated hemolysis in vivo. We also observed similar results from in vitro experiments studying pre-existing anti-SSL7 antibodies in humans. In addition, we showed that ocular AC inoculation of SSL7 prior to SSL7 administration significantly inhibited the production of SSL7-specific antibodies in vivo.

SSL7, encoded by the genome of the pathogen Staphylococcus aureus, was discovered more than 10 years ago to be able to bind to both IgA and C5, thereby inhibiting IgA-FcaRI binding and complement killing of the pathogen in in vitro assays[16, 28]. SSL7 is a small protein $(\sim 17 \mathrm{KDa})$ and a potent $\mathrm{C} 5$ inhibitor, working at concentrations as low as 0.3 $\mathrm{nM}[16]$. Given the strong potency of SSL7 in inhibiting C5, it should hold promise as a new alternative to eculizumab for treating MAC-mediated diseases such as PNH and CAD. However, partially because of its predicted immunogenicity in vivo, there has been no report on the in vivo evaluation of the efficacy of SSL7 in preventing complement-mediated hemolysis. Encouraged by the recent Phase II clinical trial (NCT02591862) on Coversin, a foreign complement inhibitor produced by the soft tick, we evaluated the potential of SSL7 using a previously established complement-mediated intravascular hemolysis model in mice[22, 23]. Injection of human NHS (complement) in mice causes rapid and massive hemolysis of the mouse erythrocytes intravascularly that can be measured by assessing hematocrit reduction, plasma hemoglobin levels and/or hemoglobinuria severity. Massive hemolysis is caused by activated human complement in mice, thus inhibiting human C5 efficiently controls the lysis of mouse erythrocytes. Our data that administration of SSL7 as low as $2 \mu \mathrm{g} /$ mouse significantly inhibited the complement-mediated intravascular hemolysis and the development of hemoglobinuria clearly demonstrated that SSL7 is effective in inhibiting human complement-mediated hemolysis in vivo. These data suggest that SSL7 has the potential to be developed as an alternative to eculizumab, especially for the treatment of acute MAC-mediated intravascular hemolysis in certain catastrophic conditions such as drug-induced immune hemolytic anemia and $\mathrm{ABO}$-incompatible red blood cell transfusions.

However, SSL7 is a foreign protein, which raises immunogenicity issues for treatment purposes. In addition, SSL7 has been found to interact with dendritic cells and other leukocytes[29], which could enhance its immunogenicity in vivo. If anti-SSL7 antibodies are elicited, it is therefore predicted that these antibodies would quickly neutralize the administered SSL7, rendering it ineffective. Our data indicate that although SSL7 does have immunogenicity, this issue was not as serious as it was initially thought. When injecting mice with SSL7 multiple times to mimic repetitive treatments, we found that it did raise anti-SSL7 antibodies but did not significantly reduce the effect of SSL7 in inhibiting complement-mediated hemolysis in vivo. More importantly, we found that pre-existing antiSSL7 antibodies in humans did not significantly reduce the complement-inhibitory activity of SSL7 either. In these human sample studies, to avoid the potential interferences induced by different complement activities in sera from different donors, we first heat-inactivated the 
endogenous complement in the sera before mixing them with SSL7 to allow the interaction between SSL7 and anti-SSL7 antibodies in the sera, then testing the mixtures in a standard hemolysis inhibition assay. This heat treatment is known to inactivate complement but has no effect on antibody activities, therefore, doing so should minimize the unwanted variables induced by different complement activities in different sera. However, like antibodies, factor $\mathrm{H}$, an abundant and potent complement inhibitor in the serum, is also stable in the heatinactivation process. The difference of factor $\mathrm{H}$ activities in the heat-inactivated sera from different donors could also complicate the sequential complement assays. To address this issue, inspired by our recent reports showing that MSCs are damaged by complement after contact with serum[21] and that painting complement inhibitors such as factor $\mathrm{H}[25$ ] or heparin[24] effectively protects MSC from the complement-mediated cell injury, we painted SSL7 on MSC surface, then allowed the anti-SSL7 antibodies in different sera to bind to the SSL7 without activating complement in these serum samples (because complement activities in the serum from each donor would be different). We then used a pooled NHS as the source of complement for all the MSC samples to allow a reliable comparison of the protective effects of the SSL7 in the presence of different titers of anti-SSL7 antibodies. In addition, because different levels of anti-SSL7 immune complexes on the MSC surface might initiate various degrees of complement activation through the classical pathway, which can complicate our data interpretation, we used the GVB-Mg-EGTA buffer in our assays to allow only the alternative pathway of complement activation occurs after MSCs contact serum. Our data demonstrated that even in the presence of anti-SSL7 antibodies developed in mice after repetitive SSL7 administrations, or in humans potentially after Staphylococcus aureus infection(s), exogenously administered SSL7 was still able to efficiently inhibit complement-mediated hemolysis or MSC damage. The precise mechanism of this effect was not ascertained, but low affinities of these anti-SSL7 antibodies, preference of binding epitopes irrelevant to SSL7 complement inhibitory activity and/or the high potency of SSL7 in inhibiting complement are possible explanations. These results suggest that SSL7, even with its existing immunogenicity, could still be used repeatedly for the treatment of MACmediated diseases and to improve current MSC-based therapies.

In addition, there are many possible ways to establish antigen-specific tolerance to a target protein including feeding the protein or related peptides[30] or infusing tolerogenic dendritic cells[31] to reduce SSL7 immunogenicity for further improving future SSL7-based therapies. We chose to test the effect of the approach termed Anterior Chamber Associated Immune Deviation (ACAID). ACAID was originally discovered by Kaplan and Sterilin more than 30 years ago[32]. It is now well-established that inoculation of a protein antigen, including allotransplantation antigens, soluble protein antigens, viral antigens, or tumor antigens, in the AC of the eye, which is an immunoprivileged organ, induces systemic tolerance of the inoculated antigen[33, 34]. Indeed, we found that prior AC inoculation of SSL7 significantly suppressed SSL7-specific antibody production after repetitive SSL7 administration. Intraocular injection of foreign proteins in patients is a common practice in the clinic as many age-related macular degeneration patients are being treated by repeat intraocular injections of anti-VEGF mAbs[35]. Our data here again suggest that SSL7 has potential to be developed as an economical alternative to eculizumab, especially after the induction of SSL7-specific tolerance in patients by different approaches such as ACAID. 
In summary, C5 is a validated target for treating many complement-mediated diseases especially diseases in which MAC is integrally involved in the pathogenesis. We demonstrated that SSL7, a $\sim 17$ kDa small protein produced by Staphylococcus aureus, even with its existing immunogenicity as a foreign protein, was still able to reduce complementmediated intravascular hemolysis in vivo in the presence of anti-SSL7 antibodies. We also showed that ACAID could be used to induce SSL7-specific immune tolerance, which reduced the production of anti- SSL7 antibodies after its repetitive administrations. Our data suggest that SSL7, as well as its derivatives, hold promise an economical alternative to eculizumab for treating many complement-mediated diseases such as complement-mediated intravascular hemolysis, especially after the combined induction of SSL7-specific immune tolerance.

\section{Acknowledgments}

This work was supported in part by NIH grants R01 AR061564 (to F.L.) and R01 DK10358 (to F.L.). The authors declare no conflict of interest. Parts of this study were previously presented in XXVI International Complement Workshop at Kanazawa, Japan, 2016[36] and in American Association of Immunologists Annual Meeting at Texas, USA, 2018[37].

\section{References}

1. Song WC, Sarrias MR, Lambris JD (2000) Complement and innate immunity. Immunopharmacology 49: 187-198 [PubMed: 10904117]

2. Meri S, Jarva H (1998) Complement regulation. Vox Sang 74 Suppl 2: 291-302 [PubMed: 9704459]

3. Ware RE, Rosse WF, Howard TA (1994) Mutations within the Piga gene in patients with paroxysmal nocturnal hemoglobinuria. Blood 83: 2418-2422 [PubMed: 8167330]

4. Hill A, DeZern AE, Kinoshita T, Brodsky RA (2017) Paroxysmal nocturnal haemoglobinuria. Nat Rev Dis Primers 3: 17028 DOI 10.1038/nrdp.2017.28 [PubMed: 28516949]

5. Chapin J, Terry HS, Kleinert D, Laurence J (2016) The role of complement activation in thrombosis and hemolytic anemias. Transfus Apher Sci 54: 191-198. DOI 10.1016/j.transci.2016.04.008 [PubMed: 27156108]

6. Berentsen S (2016) Cold agglutinin disease. Hematology Am Soc Hematol Educ Program 2016: 226-231. DOI 10.1182/asheducation-2016.1.226 [PubMed: 27913484]

7. Hillmen P, Young NS, Schubert J, Brodsky RA, Socie G, Muus P, Roth A, Szer J, Elebute MO, Nakamura R, Browne P, Risitano AM, Hill A, Schrezenmeier H, Fu CL, Maciejewski J, Rollins SA, Mojcik CF, Rother RP, Luzzatto L (2006) The complement inhibitor eculizumab in paroxysmal nocturnal hemoglobinuria. N Engl J Med 355: 1233-1243. DOI 10.1056/NEJMoa061648 [PubMed: 16990386]

8. Hillmen P, Hall C, Marsh JC, Elebute M, Bombara MP, Petro BE, Cullen MJ, Richards SJ, Rollins SA, Mojcik CF, Rother RP (2004) Effect of eculizumab on hemolysis and transfusion requirements in patients with paroxysmal nocturnal hemoglobinuria. N Engl J Med 350: 552-559. DOI 10.1056/ NEJMoa031688 [PubMed: 14762182]

9. Roth A, Huttmann A, Rother RP, Duhrsen U, Philipp T (2009) Long-term efficacy of the complement inhibitor eculizumab in cold agglutinin disease. Blood 113: 3885-3886. DOI 10.1182/ blood-2009-01-196329 [PubMed: 19372265]

10. Shapiro R, Chin-Yee I, Lam S (2015) Eculizumab as a bridge to immunosuppressive therapy in severe cold agglutinin disease of anti-Pr specificity. Clin Case Rep 3: 942-944. DOI 10.1002/ ccr3.399 [PubMed: 26576277]

11. Coyle D, Cheung MC, Evans GA (2014) Opportunity cost of funding drugs for rare diseases: the cost-effectiveness of eculizumab in paroxysmal nocturnal hemoglobinuria. Med Decis Making 34: 1016-1029. DOI 10.1177/0272989X14539731 [PubMed: 24990825] 
12. Nunn MA, Sharma A, Paesen GC, Adamson S, Lissina O, Willis AC, Nuttall PA (2005) Complement inhibitor of C5 activation from the soft tick Ornithodoros moubata. J Immunol 174:2084-2091 [PubMed: 15699138]

13. Soltys J, Kusner LL, Young A, Richmonds C, Hatala D, Gong B, Shanmugavel V, Kaminski HJ (2009) Novel complement inhibitor limits severity of experimentally myasthenia gravis. Ann Neurol 65: 67-75. DOI 10.1002/ana.21536 [PubMed: 19194881]

14. Lin F, Kaminski HJ, Conti-Fine BM, Wang W, Richmonds C, Medof ME (2002) Markedly enhanced susceptibility to experimental autoimmune myasthenia gravis in the absence of decayaccelerating factor protection. J Clin Invest 110: 1269-1274. DOI 10.1172/JCI16086 [PubMed: 12417565]

15. Kaminski HJ, Kusner LL, Richmonds C, Medof ME, Lin F (2006) Deficiency of decay accelerating factor and CD59 leads to crisis in experimental myasthenia. Exp Neurol 202: 287293. DOI 10.1016/j.expneurol.2006.06.003 [PubMed: 16859686]

16. Langley R, Wines B, Willoughby N, Basu I, Proft T, Fraser JD (2005) The staphylococcal superantigen-like protein 7 binds IgA and complement C5 and inhibits IgA-Fc alpha RI binding and serum killing of bacteria. J Immunol 174: 2926-2933 [PubMed: 15728504]

17. Laursen NS, Gordon N, Hermans S, Lorenz N, Jackson N, Wines B, Spillner E, Christensen JB, Jensen M, Fredslund F, Bjerre M, Sottrup-Jensen L, Fraser JD, Andersen GR (2010) Structural basis for inhibition of complement C5 by the SSL7 protein from Staphylococcus aureus. Proc Natl Acad Sci U S A 107: 3681-3686. DOI 10.1073/pnas.0910565107 [PubMed: 20133685]

18. Bestebroer J, Aerts PC, Rooijakkers SH, Pandey MK, Kohl J, van Strijp JA, de Haas CJ (2010) Functional basis for complement evasion by staphylococcal superantigen-like 7. Cell Microbiol 12: 1506-1516. DOI 10.1111/j.1462-5822.2010.01486.x [PubMed: 20545943]

19. Zhang L, Li Y, Payne J, Srivastava S, Fan X, Fung J, Li X, Kern TS, Lin F (2016) Presence of retinal pericyte-reactive autoantibodies in diabetic retinopathy patients. Sci Rep 6: 20341 DOI 10.1038/srep20341 [PubMed: 26839120]

20. Lorenz N, Clow F, Radcliff FJ, Fraser JD (2013) Full functional activity of SSL7 requires binding of both complement C5 and IgA. Immunol Cell Biol 91: 469-476. DOI 10.1038/icb.2013.28 [PubMed: 23797068]

21. Li Y, Lin F (2012) Mesenchymal stem cells are injured by complement after their contact with serum. Blood 120: 3436-3443. DOI 10.1182/blood-2012-03-420612 [PubMed: 22966167]

22. Zhang L, Qiu W, Crooke S, Li Y, Abid A, Xu B, Finn MG, Lin F (2017) Development of Autologous C5 Vaccine Nanoparticles to Reduce Intravascular Hemolysis in Vivo. ACS Chem Biol 12: 539-547. DOI 10.1021/acschembio.6b00994 [PubMed: 28045484]

23. Ino Y, Sato T, Suzuki S, Iwaki M, Yoshikawa T (1987) Inhibitory effects of FUT-175, a new synthetic protease inhibitor, on intravascular hemolysis by human serum in mice. Int $\mathbf{J}$ Immunopharmacol 9: 533-537 [PubMed: 3623771]

24. Li Y, Fung J, Lin F (2016) Local Inhibition of Complement Improves Mesenchymal Stem Cell Viability and Function After Administration. Mol Ther 24: 1665-1674. DOI 10.1038/mt.2016.142 [PubMed: 27377042]

25. Li Y, Qiu W, Zhang L, Fung J, Lin F (2016) Painting factor H onto mesenchymal stem cells protects the cells from complement- and neutrophil-mediated damage. Biomaterials 102: 209-219. DOI 10.1016/j.biomaterials.2016.05.055 [PubMed: 27343468]

26. Mizuno K, Clark AF, Streilein JW (1988) Induction of anterior chamber associated immune deviation in rats receiving intracameral injections of retinal $S$ antigen. Curr Eye Res 7:627-632 [PubMed: 3261228]

27. Hara Y, Caspi RR, Wiggert B, Chan CC, Streilein JW (1992) Use of ACAID to suppress interphotoreceptor retinoid binding protein-induced experimental autoimmune uveitis. Curr Eye Res 11 Suppl: 97-100 [PubMed: 1424755]

28. Ramsland PA, Willoughby N, Trist HM, Farrugia W, Hogarth PM, Fraser JD, Wines BD (2007) Structural basis for evasion of IgA immunity by Staphylococcus aureus revealed in the complex of SSL7 with Fc of human IgA1. Proc Natl Acad Sci U S A 104: 15051-15056. DOI 10.1073/pnas. 0706028104 [PubMed: 17848512] 
29. Al-Shangiti AM, Nair SP, Chain BM (2005) The interaction between staphylococcal superantigenlike proteins and human dendritic cells. Clin Exp Immunol 140: 461-469. DOI 10.1111/j. 1365-2249.2005.02789.x [PubMed: 15932507]

30. Weiner HL, da Cunha AP, Quintana F, Wu H (2011) Oral tolerance. Immunol Rev 241:241-259. DOI 10.1111/j.1600-065X.2011.01017.x [PubMed: 21488901]

31. Moreau A, Alliot-Licht B, Cuturi MC, Blancho G (2016) Tolerogenic dendritic cell therapy in organ transplantation. Transpl Int DOI 10.1111/tri.12889

32. Kaplan HJ, Streilein JW (1978) Immune response to immunization via the anterior chamber of the eye. II. An analysis of F1 lymphocyte-induced immune deviation. J Immunol 120: 689-693 [PubMed: 632581]

33. Ashour HM (2015) Immune tolerance elicited via unique ocular and oral routes. Curr Mol Med 15: 78-81 [PubMed: 25601470]

34. Niederkorn JY (2007) The induction of anterior chamber-associated immune deviation. Chem Immunol Allergy 92: 27-35. DOI 10.1159/000099251 [PubMed: 17264480]

35. Villegas VM, Aranguren LA, Kovach JL, Schwartz SG, Flynn HW Jr. (2017) Current advances in the treatment of neovascular age-related macular degeneration. Expert Opin Drug Deliv 14: $273-$ 282. DOI 10.1080/17425247.2016.1213240 [PubMed: 27434329]

36. Li Y, Clow F, Fung JJ, Fraser JD, Lin F (2016) Therapeutic potential of staphylococcal superantigen-like protein 7 for acute complement activation-mediated diseases. Immunobiology 221: 1148-1148. DOI 10.1016/j.imbio.2016.06.055

37. Li Y, Lin F (2018) Therapeutic potential of staphylococcal superantigen-like protein 7 for acute complement activation mediated diseases. The Journal of Immunology 200: 50.20-50.20 


\section{Key points:}

1. SSL7 functions in the presence of anti-SSL7 antibodies both in vitro and in vivo

2. SSL7 has the potential to be developed as a new and economical complement inhibitor for treating complement-mediated hemolysis

\section{Key messages:}

3. SSL7 functions in the presence of anti-SSL7 antibodies both in vitro and in vivo

4. SSL7 has the potential to be developed as a new and economical complement inhibitor for treating complement-mediated hemolysis 


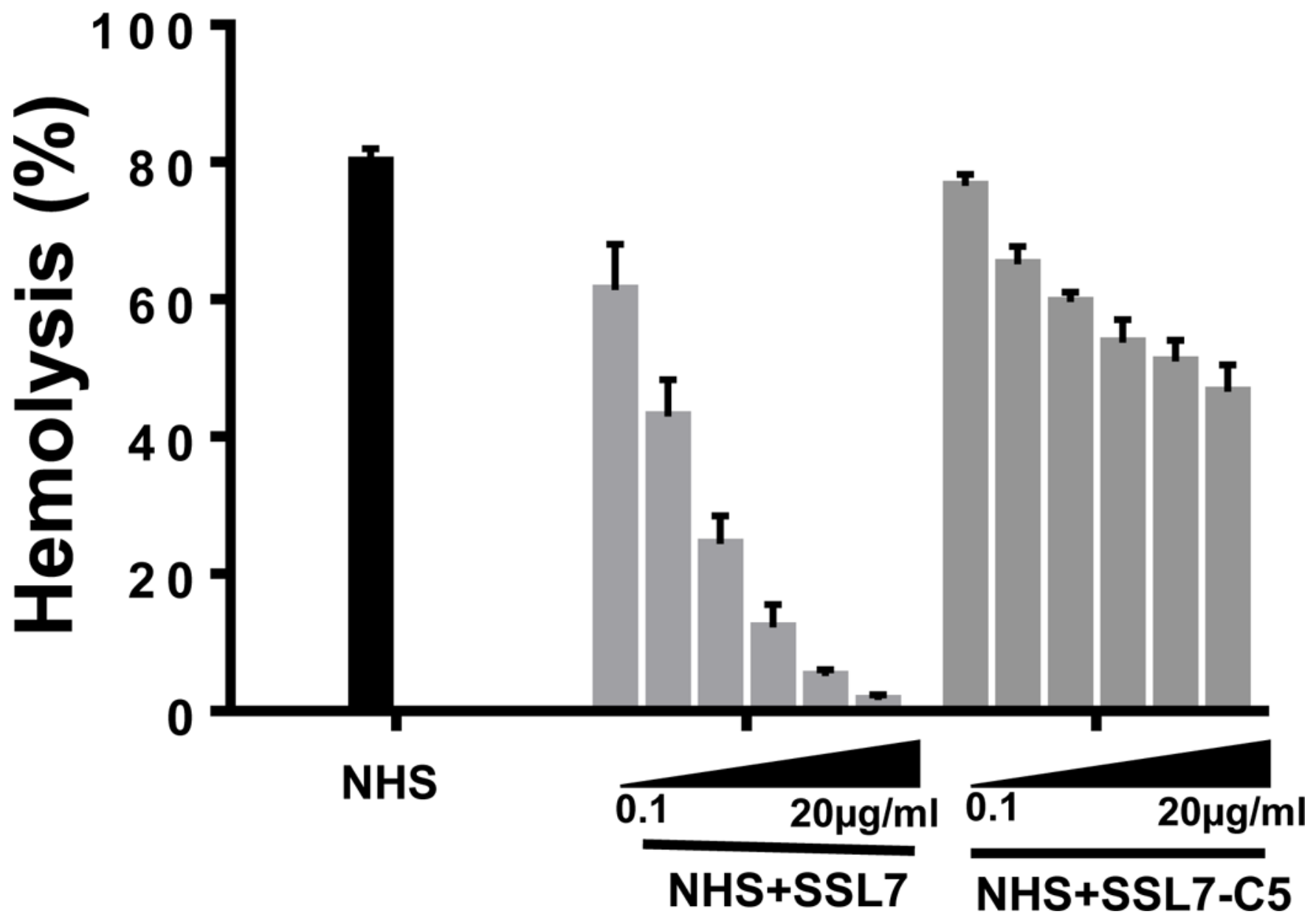

Figure 1.

SSL7, but not SSL7-C5, significantly inhibits complement-mediated hemolysis in vitro. Complement-mediated lysis of $\mathrm{E}^{\text {shA }}$ in the absence of (NHS), or in the presence of different concentrations $(0.1,0.5,1,5,10$, and $20 \mu \mathrm{g} / \mathrm{ml}$ ) of SSL7 (NHS+ SSL7) or SSL7-C5 (NHS +SSL7-C5) were analyzed. Combined results from 3 independent experiments. . $* p<0.05$ (student's t-test) 
A.

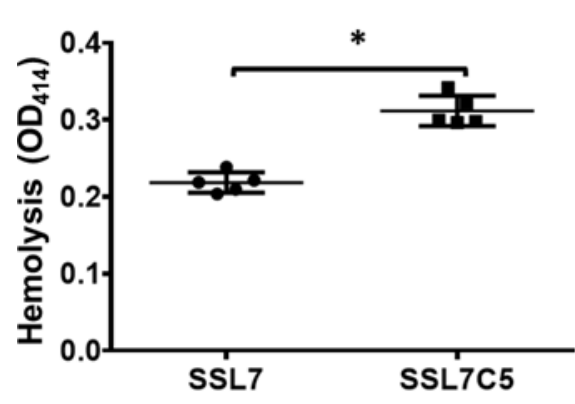

B.

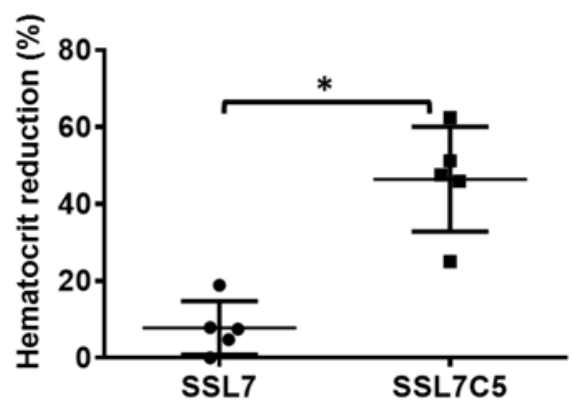

c.

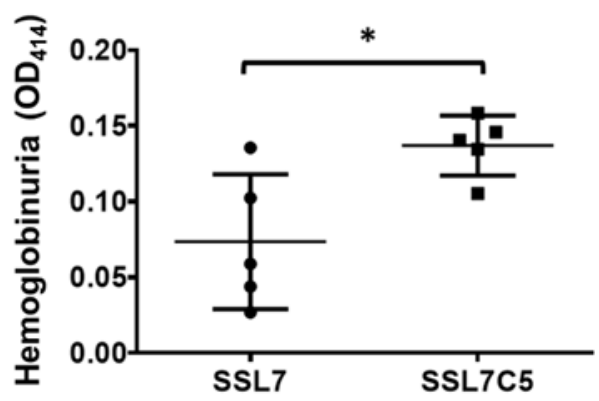

Figure 2.

SSL7 reduces complement-mediated hemolytic anemia in vivo. WT C57BL/6 mice were injected with SSL7 and NHS. Then blood and urine were collected, and the hemolysis (A) hematocrit reduction (B) and hemoglobinuria (C) were assessed. $\mathrm{n}=5$ in each group, $* p<0.05$ ( student's t-test) 

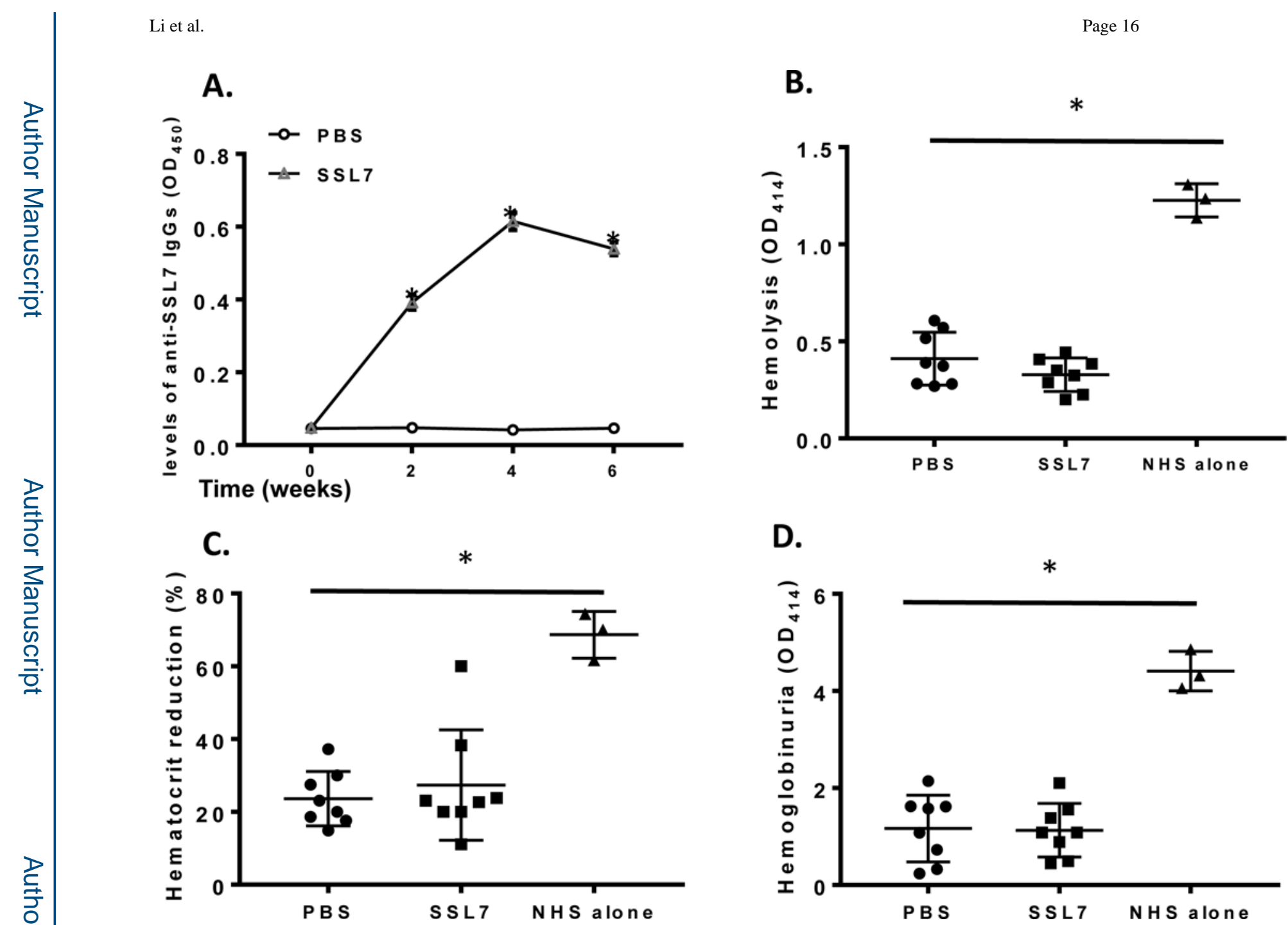

Figure 3.

Repetitive administration of SSL7 raises anti-SSL7 antibodies but does not reduce SSL7 treatment efficacy. WT C57BL/6 mice were intraperitoneally injected with SSL7 in PBS (SSL7) or PBS alone (PBS) once every other week for 6 weeks, then serum SSL7-reactive IgG levels were analyzed by ELISA ( $\mathrm{n}=8$ in each group) (A). These mice were then injected with SSL7 followed by NHS to induce hemolysis. Levels of released hemoglobin in the plasma (OD414) (B), hematocrit reduction (C) and severities of hemoglobinuria (OD414) (D) were assessed. $\mathrm{n}=8$ in each group. Naïve mice received NHS but no SSL7 treatment $(\mathrm{n}=3)$ were included as controls (NHS alone). ${ }^{*} p<0.05$ (ANOVA) 


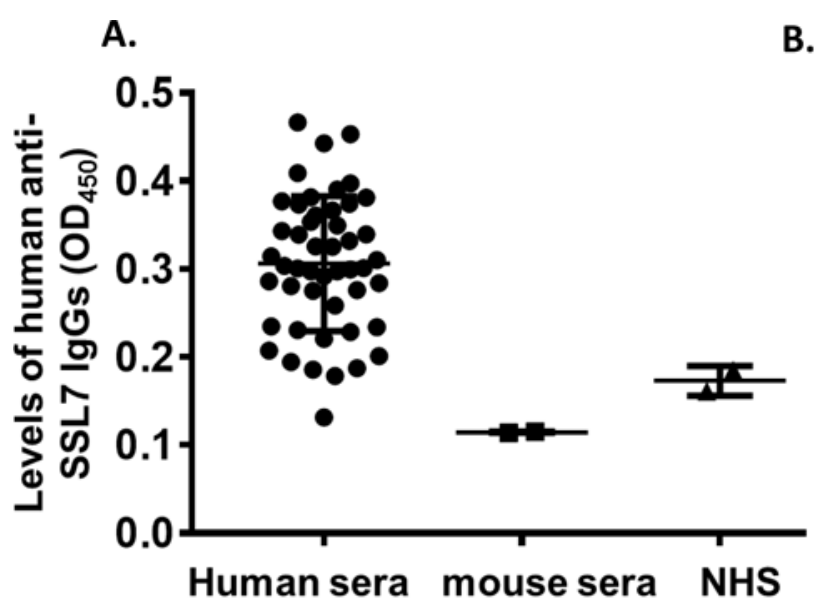

C.

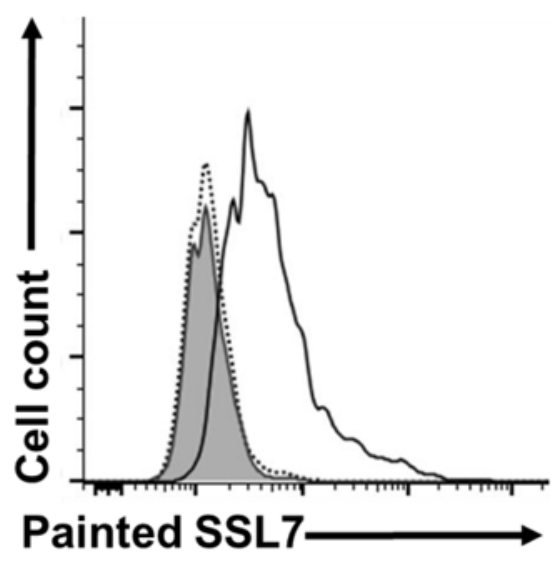

D.
B.

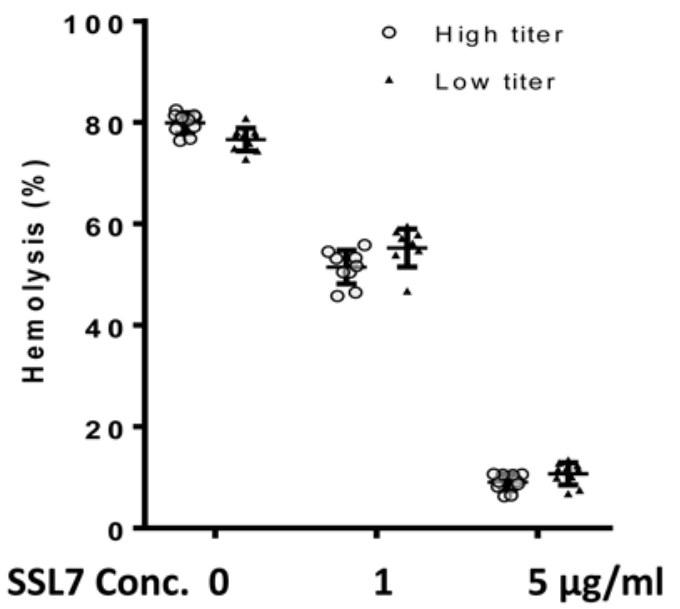

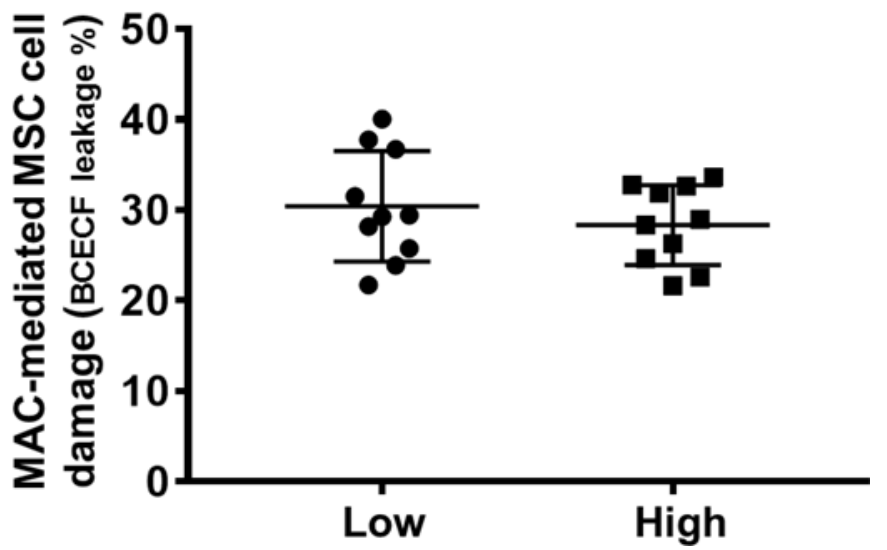

Figure 4.

SSL7-reactive antibodies pre-existing in the human sera do not significantly reduce the complement inhibitory activity of SSL7. (A) Levels of anti-SSL7 IgGs were measured by ELISA in 50 serum samples previously collected from normal human donors, showing antiSSL7 IgGs exist in some of the donors. Each dot represents one subject. Sera from 2 mice (mouse sera) were included as negative controls. Two different batches NHS (NHS) used in our complement assays as the sources of complement were also analyzed in the same assay, showing no appreciable amount of anti-SSL7 IgGs within. * ${ }^{*}<0.05$ (ANOVA) (B).

Assessment of SSL7 complement inhibitory activity in the presence of anti-SSL7 antibodies. Ten human serum samples containing high or low titers of anti-SSL7 IgGs were heatinactivated, then incubated with 0,1 or $5 \mu \mathrm{g} / \mathrm{ml}$ of SSL7. Complement inhibitory activities of the mixtures were analyzed in a conventional complement-mediated hemolysis assay. (C). Painting SSL7 onto MSCs for the measurement of its complement-inhibitory activity. SSL7 was painted onto MSCs, then the painted SSL7 on the MSCs was detected by staining the cells with an anti-SSL7 IgG followed by flow cytometric analysis. Shaded area, isotype control, dotted line, MSCs without painting; solid line, MSCs with painted SSL7. (D). The complement-inhibitory activity of SSL7 assessed by measuring its efficacy in protecting MSCs from complement (MAC)-mediated damage. MSCs painted with SSL7 were first incubated with 10 sera samples containing the highest (High) or 10 sera samples containing 
the lowest (Low) levels of anti-SSL7 IgGs in the presence of EDTA, after washing, these cells were then subjected to complement-mediated cell injury, which was quantitated by measuring levels of BCECF leakages. * $p<0.05$ (student's t-test) 
A.

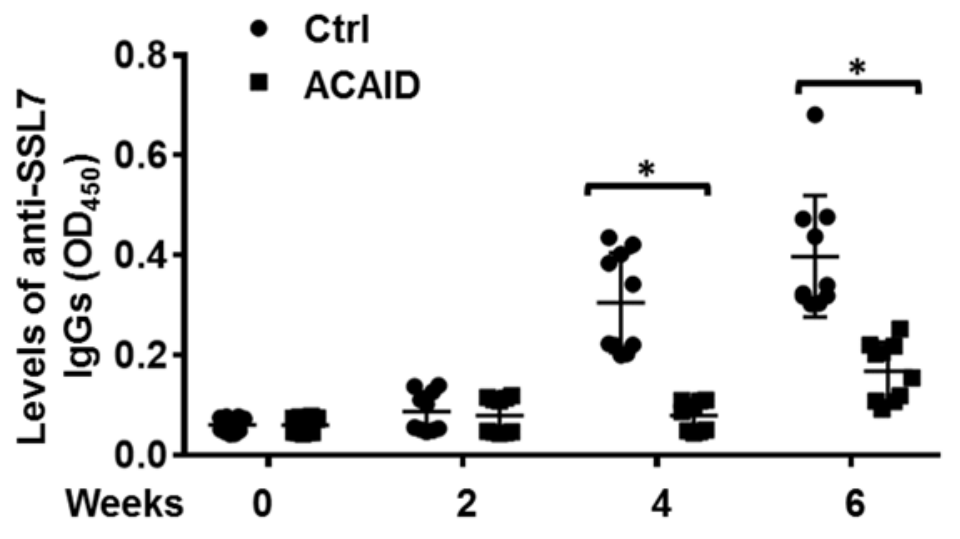

C.

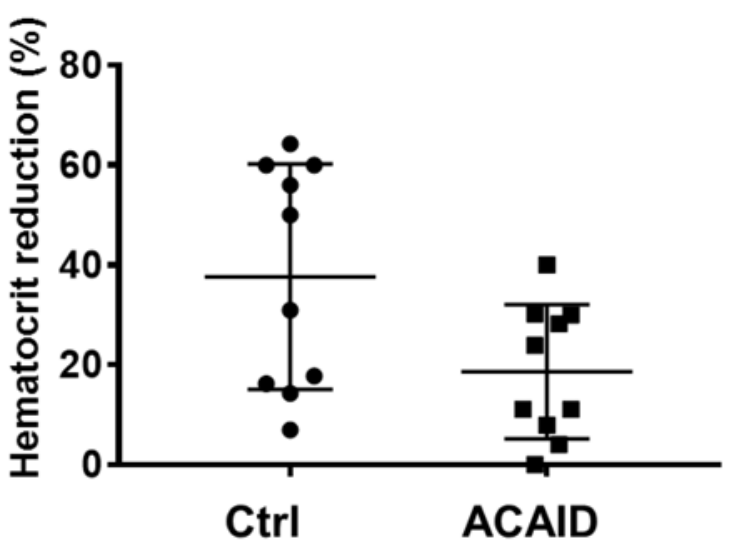

B.

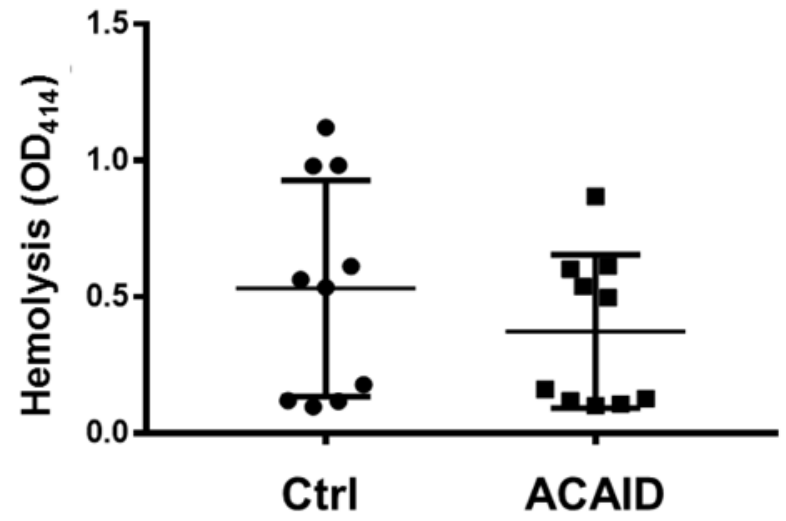

D.

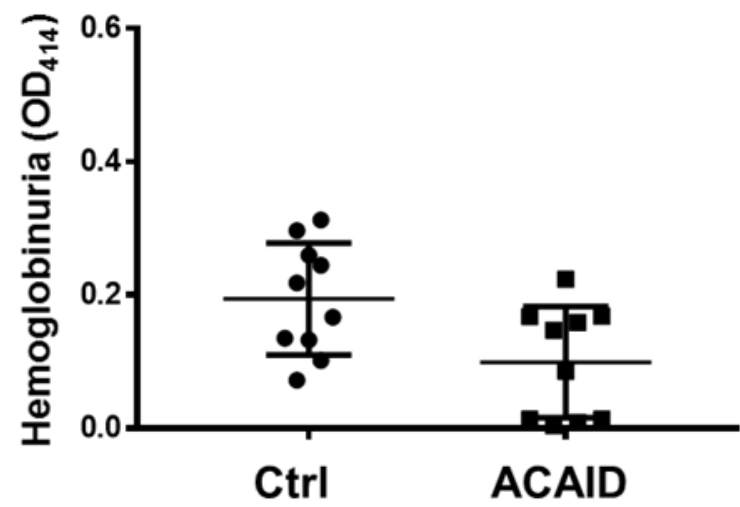

Figure 5.

Ocular anterior chamber inoculation of SSL7 reduces anti-SSL7 antibody production after repetitive intraperitoneal injection. SSL7 in PBS (ACAID) or PBS alone (Ctrl) was first injected into the anterior chambers of each of the WT C57BL/6 mice, then 2 weeks later, these mice were treated by repetitive intraperitoneal injection of SSL7 every other week for 6 weeks. Levels of SSL7-specific IgGs in the plasma of these mice at different time points were analyzed by ELISA (A). Following this, these mice were injected with NHS and the efficacy of SSL7 in reducing complement-mediated hemolysis was assessed by measuring the intravascular hemolysis (B), hematocrit reduction (C) and hemoglobinuria (D). $n=10$ in each group, * $p<0.05$ (student's $t$-test) 